\title{
A DESCENTRALIZAÇÃO DO SINASC E A COMPLETUDE DAS VARIÁVEIS DA DECLARAÇÃO DE NASCIDO VIVO EM MUNICÍPIOS MINEIROS DE 1998 A 2005
}

\author{
THE SINASC DESCENTRALIZATION AND THE \\ COMPLETENESS OF VARIABLES ON BIRTH \\ CERTIFICATES, IN MUNICIPALITIES OF MINAS \\ GERAIS FROM 1998 TO 2005
}
Eliete Albano de Azevedo Guimarães ${ }^{1}$, Antônio Ignácio de Loyola Filho ${ }^{2}$, Zulmira Maria de Araújo Hartz ${ }^{3}$, Antônio José de Meira ${ }^{4}$, Zélia Maria Profeta Luz ${ }^{5}$

\begin{abstract}
RESUMO
O Sistema de Informações Sobre Nascidos Vivos (SINASC) foi descentralizado com a finalidade de aprimorar a qualidade da informação e sua utilização, subsidiando a avaliação de serviços e os estudos epidemiológicos. Este estudo avaliou a completude das variáveis relativas ao recém-nascido, à mãe e à gestação e parto presentes na declaração de nascimento, em 38 municípios no estado de Minas Gerais. Foram utilizados os registros de nascidos vivos de mães residentes, disponível na base de dados do Sistema, entre os anos de 1998 e 2005. A avaliação levou em consideração o porte populacional e a condição de gestão dos municípios. Definiu-se como indicador o percentual de campos não preenchidos, e o padrão de qualidade adotado foi o definido por Mello Jorge et al. (1996). Valores entre $90 \%$ e $100 \%$ foram considerados adequados; de $70 \%-89,9 \%$ não adequados, e crítico quando estes eram menores que $70 \%$. Observou-se aprimoramento no preenchimento da DNV ao longo do período analisado, no conjunto dos municípios avaliados. Verificou-se ainda redução importante da incompletude nas variáveis raça/cor, grau de instrução, estado civil, filhos nascidos vivos e nascidos mortos. A descentralização do Sistema de Informações Sobre Nascidos Vivos possibilitou a melhoria da completude das informações nos municípios estudados, independentemente do porte e da condição de habilitação.
\end{abstract}

Palavras-chave: declaração de nascimento; nascidos vivos.

1 Doutoranda do Curso de Pós-Graduação em Ciências da Saúde do Centro de Pesquisas René Rachou, Fundação Oswaldo Cruz - CPqRR/FIOCRUZ. Belo Horizonte, Minas Gerais. Professora do Curso de Enfermagem do Campus Centro Oeste da Universidade Federal de São João Del Rei, Minas Gerais.

2 Pesquisador do Centro de Pesquisas René Rachou, Fundação Oswaldo Cruz -CPqRR/FIOCRUZ. Belo Horizonte, Minas Gerais. Professor do Departamento de Enfermagem Aplicada da Escola de Enfermagem da Universidade Federal de Minas Gerais.

3 Pesquisadora da Escola Nacional de Saúde Pública, Fundação Oswaldo Cruz/ENSP/FIOCRUZ. Manguinhos, Rio de Janeiro.

4 Diretor da Gerência de Inteligência Epidemiológica da Secretaria de Estado de Saúde de Minas Gerais. Cidade Administrativa, Belo Horizonte, MG.

5 Pesquisadora do Centro de Pesquisas René Rachou, Fundação Oswaldo Cruz -CPqRR/FIOCRUZ. Belo Horizonte, Minas Gerais.

Correspondência para: eguimaraes@cpqrr.fiocruz.br

Como citar este artigo: Guimarães EAA, et al. The SINASC descentralization and the completeness of variables on Birth Certificates, in municipalities of Minas Gerais from 1998 to 2005. Journal of Human Growth and Development 2011; 21(3): 832-840.

Artigo submetido em 06.03.10, aceito em 30.08.11. 


\begin{abstract}
Live Birth Information System (SINASC) was decentralized in order to improve the use and the quality of the information, being the basis for service evaluation and epidemiological studies. This study evaluated the percentage of unfilled fields in the variables related to newborn, mother and delivery on Live Birth Certificates in 38 municipalities of Minas Gerais. Population was composed by the newborns of resident mothers, available at the database, between 1998 and 2005. The evaluation considered the population size and the healthcare management of studied municipalities. It was observed an improvement in the filling of Live Birth Certificates in the analyzed period in the studied municipalities. An important reduction on the percentage of unfilled fields was observed in the variables: race/color, literacy, civil state, number of live born sons and number of dead born sons. Live Birth Information System decentralization led to an improvement in the percentage of unfilled fields of certificates in the studied municipalities, regardless of population size and healthcare management.
\end{abstract}

Key words: birth certificates; live births.

\section{INTRODUÇÃO}

A Constituição Federal de 1988 foi o marco de intensas transformações ocorridas no sistema de saúde brasileiro, pois consolidou mudanças importantes no modelo político-institucional. Reconheceu a saúde como um direito a ser assegurado pelo Estado e garantiu a municipalização como um princípio para estruturar as ações e serviços de saúde ${ }^{1}$.

A descentralização dos serviços de saúde envolve não só mudanças no modelo assistencial, mas também possibilita a criação de uma rede de relações e a ampliação da participação dos cidadãos na tomada de decisões, o que contribui para a democratização da gestão das políticas públicas de saúde ${ }^{1-2}$. Entendese que esse processo é gradual e que perpassa um campo com inúmeros obstáculos e contradições².

As Leis Orgânicas da Saúde (Lei $8080 / 1990$ e Lei $8142 / 1990$ ) e as Normas Operacionais Básicas (NOB-SUS 1991, 1993, 1996; NOAS-SUS 2001 e 2002) redefiniram os papéis institucionais, a co-responsabilização entre as esferas de governo, a forma de gestão do sistema, seu ordenamento e controle e o repasse de recursos para as ações coletivas e individuais. Além disso, estes instrumentos normativos têm estabelecido regras e metas que colocam os municípios brasileiros frente à necessidade de organizar as informações em saúde pro- duzidas ao nível local, que possibilitem responder com maior agilidade e especificidade a nova lógica gerencial e o redirecionamento do setor. Os sistemas de informação em saúde constituíramse como instâncias de governabilidade e de negociação entre gestores dos três níveis de governo ${ }^{3-4}$.

O SINASC - Sistema de Informações Sobre Nascidos Vivos, implantado em 1990 pelo Ministério da Saúde, foi descentralizado para todos os municípios. A fonte de dados do SINASC é a Declaração de Nascidos Vivos (DNV), documento individualizado que deve ser preenchido para todos os nascimentos vivos ocorridos no país, sejam eles hospitalares ou domiciliares 3,5 .

O Sistema surge, sobretudo, para aprimorar as informações referentes ao recém-nascido, à mãe e às condições de nascimento ${ }^{6}$ e possibilitar também o monitoramento da qualidade dos dados produzidos, em termos da cobertura7, confiabilidade $^{8-9}$ e completude das informações ${ }^{10-12}$. Variáveis como peso ao nascer, duração da gestação, tipo de parto, idade e grau de instrução maternos e paridade são utilizadas na construção de indicadores de saúde e demográficos de uma população, sendo ainda avaliados na pactuação da atenção básica e na programação das ações de vigilância em saúde ${ }^{13}$. Embora seja um sistema específico, as informações do SINASC, quando acessíveis aos gestores e à comuni- 
dade, podem contribuir para o planejamento das ações de saúde voltadas para esse segmento populacional ${ }^{13}$.

Apesar dos avanços normativos institucionais e da política de informação em saúde, a descentralização do SINASC vem apresentando entraves na sua operacionalização, principalmente em relação à efetividade das suas informações ${ }^{13}$.

Para alguns municípios do estado de Minas Gerais (MG), a implantação do SINASC teve início em 1994, mas para a maioria deles, a descentralização iniciouse a partir de $1998^{14}$. Apesar da melhora da qualidade das informações no Estado, ainda há lacunas no processo informacional, em particular na coleta, no processamento e na utilização dos dados ${ }^{14-16}$. Variações na cobertura e na qualidade dos dados ainda são verificadas, o que pode estar relacionado com a forma diferenciada com a qual se deu a descentralização do sistema nos municípios.

Nesse contexto, estudos avaliativos são fundamentais, possibilitando determinar o valor ou o significado de uma atividade, programa ou política, assim como corrigir ou melhorar ações com o propósito de aumentar a pertinência, eficácia e eficiência das atividades de saú$\mathrm{de}^{17}$. Assim, o objetivo é prover avaliação normativa sobre a qualidade da completude da Declaração de Nascido Vivo em municípios de Minas Gerais no período de 1998 a 2005.

\section{MÉTODO}

O presente trabalho é uma avaliação normativa sobre a qualidade da completude das Declarações de Nascidos Vivos em municípios de MG de 1998 a 2005. A avaliação é considerada normativa quando é realizada com base nas definições de normas e critérios ${ }^{17,18}$. Os critérios utilizados para avaliar o grau de preenchimento da DNV no presente estudo seguiram os parâmetros propostos por Melo Jorge et al. ${ }^{6}$.

De acordo com o Plano Diretor de Regionalização de Minas Gerais (PDR), o estado está dividido em 13 Macrorregiões de Saúde, consideradas a base territorial de planejamento da atenção à saúde, em função das características demográficas, socioeconômicas, geográficas, sanitárias, epidemiológicas, oferta de serviços e relações entre municípios. Essas macrorregiões são divididas em 75 microrregiões que abrangem o universo de 853 municípios distribuídos em 28 Gerências Regionais de Saúde, que se diferenciam no nível sócio-sanitário, porte e forma de gestão ${ }^{19}$.

Para fins deste estudo, inicialmente foram selecionados a partir do Cadastro Nacional de Estabelecimento de Saúde (CNES), 394 municípios mineiros com estabelecimentos de saúde onde ocorrem partos. Estes foram classificados segundo os seguintes parâmetros: a localização regional conforme o $\operatorname{PDR}^{19}$ e o porte populacional.

Com base no número de habitantes, três categorias de porte populacional foram consideradas pelos autores: municípios com população menor de 10.000 habitantes, considerados de pequeno porte; municípios com população 10.000 a 49.999 habitantes, de médio porte e municípios com população acima de 50.000 habitantes, considerados os de grande porte.

\section{Definição dos municípios avaliados}

Para a avaliação foram selecionados aleatoriamente 38 municípios, dentre os 394, sendo três municípios de cada macrorregião de saúde, sendo um município para cada porte em cada uma das macrorregionais; com exceção apenas para a de Jequitinhonha, onde foram sorteados dois municípios (médio e pequeno porte), pois, nesta região não há os de grande porte. Estes foram classificados segundo a condição de habilitação no Sistema Único de Saúde (SUS), sendo 24 municípios habilitados na Gestão Plena da Atenção Básica (GPAB) e 14 habilitados na Gestão Plena do Sistema Municipal (GPSM).

\section{Coleta de dados}

Os dados foram obtidos por meio de CD-Rom com o consolidado das informações sobre os nascidos vivos correspondente ao período de 1998 a 2005, 
disponibilizado pela Secretaria de Vigilância em Saúde do Ministério da Saú$\mathrm{de}^{20,21}$.

A população estudada foi composta pelo total de registros de nascidos vivos de mães residentes nos municípios investigados, entre os anos de 1998 e 2005. Os anos de 1998 e 1999 marcaram o início da descentralização do SINASC para os municípios de Minas Gerais, em razão disso, neste estudo, consideramos que os anos de 2000 e 2005 correspondem ao período pós-descentralização ${ }^{14}$.

\section{Análise dos dados}

Com base nas informações da declaração de nascimento vivo, foram analisadas variáveis relativas à criança (sexo, peso ao nascer, raça/cor, Apgar $1^{\circ}$ minuto e Apgar $5^{\circ}$ minuto), a atributos maternos (idade, grau de instrução, estado civil, número de filhos nascidos vivos e número de filhos nascidos mortos) e à gestação e ao parto (duração da gestação, número de consultas de pré-natal, tipo de gravidez e tipo de parto). Os conceitos e definições adotados em cada variável foram os preconizados pelo Ministério da Saúde, segundo o manual de instruções para o preenchimento da DNV5.

Para analisar a incompletude das variáveis definiu-se como indicador o percentual de campos não preenchidos, sendo estes os campos em branco e os campos com o código 9 (ignorado) ${ }^{5}$. Apesar da pouca clareza metodológica do manual de preenchimento do SINASC ${ }^{5}$, essa foi a opção para a análise da completude das variáveis em face de dificuldades ope- racionais, uma vez que a tabulação dos dados disponibilizados pelo Ministério da Saúde não permite que se diferenciem as variáveis que não foram preenchidas (em branco) daquelas que eram realmente ignoradas pelo informante ${ }^{11}$.

O critério de qualidade adotado foi o definido por Mello Jorge et al. ${ }^{6}$, que se baseia no percentual de incompletude de preenchimento de campos da DNV (preenchimento como ignorado/branco). O preenchimento é considerado excelente quando esses percentuais não ultrapassem $10,0 \%$, é considerado bom quando os percentuais situam-se entre $10,0 \%$ a $29,9 \%$ e é considerado mau quando esse percentual supera os $30,0 \%$.

Calculou-se a média do percentual de incompletude de cada variável para o conjunto de municípios, de acordo com o porte populacional e condição de habilitação no SUS.

Com base nos percentuais de preenchimento observados, foram atribuídos escores de pontuação para cada uma das 14 variáveis. Assim, as variáveis com excelente qualidade de preenchimento receberam dez pontos, as de boa qualidade receberam cinco pontos, e as de má qualidade receberam escore dois pontos. O cálculo do grau de preenchimento da DNV foi obtido pelo percentual representado pelo somatório dos valores encontrados para cada uma das variáveis, em relação à pontuação máxima alcançável na variável. Valores entre $90 \%$ e $100 \%$ foram considerados adequados; de $70 \%$ - 89,9\% não adequados, e crítico quando estes eram menores que $70 \%$ (quadro 1).

Quadro 1: Matriz de análise e julgamento: critério e ponto de corte adotados na definição do Grau de Preenchimento das variáveis da Declaração de Nascido Vivo.

\footnotetext{
Pergunta Avaliativa ${ }^{1}$

A incompletude das variáveis tem diminuído se considerados o porte populacional e a condição de gestão do município?

Indicador

Percentual de campos em branco ou ignorado

Padrão (Melo Jorge et al.,1996)

Excelente < 10\% Bom e" $10 \%$ d" 29\%Mau e" 30\%

Ponto de corte

$<10 \%=10$ pontos

$\mathrm{e}^{\prime \prime} 10 \%, \mathrm{~d}$ " $29 \%=7$ pontos

$\mathrm{e}^{\prime \prime} 30 \%=0$ pontos

Valor observado

Refere-se ao critério/indicador coletado pelo estudo

Valor atribuído

Refere-se ao valor que será atribuído ao observado de acordo com os pontos de corte estabelecidos

Classificação do grau de preenchimento ${ }^{3}$ Refere-se à categorização na qual o item estudado será enquadrado

1 Foram avaliadas 14 variáveis

2 Ponto de corte: diz respeito ao intervalo aceitável para cada variável estudada.

3 O cálculo do grau de preenchimento da DNV foi obtido pelo percentual que o somatório dos valores encontrados para cada uma das variáveis representa em relação à pontuação máxima por critério estabelecido
} 
Para a análise dos dados foi utilizado o programa Microsoft Office EXCEL 2007.

Este estudo foi aprovado pelo Comitê de Ética do Centro de Pesquisa René Rachou/FIOCRUZ, número de registro 0001.0245.000-09.

\section{RESULTADOS}

Com a descentralização do SINASC, verificou-se para o conjunto dos municípios, indiferente do porte e do tipo de gestão, preenchimento adequado ( $90 \%$ - 100\%) para a maioria das variáveis presentes na DNV (Tabelas 1, 2).

Tabela 1: Grau de preenchimento (GP) da Declaração de Nascido Vivo dos municípios segundo o porte dos municípios no início da descentralização do SINASC (GP1: 1998 a 1999) e após (GP2: 2000 a 2005)

\begin{tabular}{|c|c|c|c|c|c|c|}
\hline \multirow{2}{*}{ Variáveis } & \multicolumn{2}{|c|}{$\begin{array}{c}\text { Pequeno } \\
(<10.000)\end{array}$} & \multicolumn{2}{|c|}{$\begin{array}{c}\text { Médio } \\
\text { (10.000 a 49.999) }\end{array}$} & \multicolumn{2}{|c|}{$\begin{array}{c}\text { Grande } \\
(>50.000)\end{array}$} \\
\hline & GP1 & GP2 & GP1 & GP2 & GP1 & GP2 \\
\hline \multicolumn{7}{|l|}{ Recém nascido } \\
\hline Peso ao nascer & 100,0 & 100,0 & 100,0 & 100,0 & 100,0 & 100,0 \\
\hline Sexo & 100,0 & 100,0 & 100,0 & 100,0 & 100,0 & 100,0 \\
\hline Apgar $1^{0}$ minuto & 96,2 & 96,2 & 80,0 & 86,2 & 89,2 & 93,3 \\
\hline Apgar 50 minuto & 100,0 & 92,3 & 82,3 & 86,2 & 89,2 & 89,2 \\
\hline $\begin{array}{l}\text { Raça/cor } \\
\text { Mãe }\end{array}$ & 20,0 & 96,2 & 20,0 & 92,3 & 20,0 & 85,0 \\
\hline Idade & 100,0 & 100,0 & 96,2 & 100,0 & 100,0 & 100,0 \\
\hline Grau de instrução & 20,0 & 100,0 & 20,0 & 100,0 & 22,5 & 95,8 \\
\hline Estado civil & 20,0 & 96,2 & 20,0 & 100,0 & 20,0 & 95,8 \\
\hline Filhos nascidos vivos & 60,0 & 76,2 & 43,1 & 72,3 & 57,5 & 91,7 \\
\hline $\begin{array}{l}\text { Filhos nascidos mortos } \\
\text { Gestação e Parto }\end{array}$ & 45,4 & 73,8 & 47,7 & 83,8 & 45,0 & 91,7 \\
\hline Tipo de gravidez & 100,0 & 100,0 & 100,0 & 100,0 & 100,0 & 100,0 \\
\hline Duração da gestação & 100,0 & 100,0 & 96,2 & 100,0 & 93,3 & 100,0 \\
\hline Consulta pré natal & 90,0 & 100,0 & 93,8 & 100,0 & 91,7 & 100,0 \\
\hline Tipo de parto & 100,0 & 100,0 & 100,0 & 100,0 & 100,0 & 100,0 \\
\hline
\end{tabular}

Tabela 2: Grau de preenchimento (GP) da Declaração de Nascido Vivo dos municípios segundo a condição de gestão dos municípios, no início da descentralização do SINASC (GP1: 1998 a 1999) e após (GP2: 2000 a 2005)

\begin{tabular}{|c|c|c|c|c|}
\hline \multirow{3}{*}{ Variáveis } & \multicolumn{4}{|c|}{ Condição de Habilitação Municipal } \\
\hline & \multicolumn{2}{|c|}{ GPAB } & \multicolumn{2}{|c|}{ GPSM } \\
\hline & GP1 & GP2 & GP1 & GP2 \\
\hline \multicolumn{5}{|l|}{ Recém nascido } \\
\hline Peso ao nascer & 100,0 & 100,0 & 100,0 & 100,0 \\
\hline Sexo & 100,0 & 100,0 & 100,0 & 100,0 \\
\hline Apgar 10 minuto & 83,8 & 89,2 & 96,4 & 96,5 \\
\hline Apgar 50 minuto & 89,2 & 87,1 & 92,9 & 92,9 \\
\hline Raça/cor & 20,0 & 93,8 & 20,0 & 87,1 \\
\hline \multicolumn{5}{|l|}{ Mãe } \\
\hline Idade & 97,9 & 100,0 & 100,0 & 100,0 \\
\hline Grau de instrução & 20,0 & 100,0 & 22,1 & 96,4 \\
\hline Estado civil & 20,0 & 100,0 & 20,0 & 92,9 \\
\hline Filhos nascidos vivos & 57,9 & 82,9 & 50,0 & 85,0 \\
\hline Filhos nascidos mortos & 47,5 & 77,5 & 39,3 & 81,4 \\
\hline \multicolumn{5}{|l|}{ Gestação e Parto } \\
\hline Tipo de gravidez & 100,0 & 100,0 & 100,0 & 100,0 \\
\hline Duração da gestação & 96,7 & 100,0 & 96,4 & 100,0 \\
\hline Consulta pré natal & 91,3 & 100,0 & 92,9 & 100,0 \\
\hline Tipo de parto & 100,0 & 100,0 & 100,0 & 100,0 \\
\hline
\end{tabular}

GPAB = Gestão Plena da Atenção Básica.

$\mathrm{GPAB}=$ Gestão Plena do Sistema Municipal. 
Ao avaliar o porte dos municípios, todas as variáveis relacionadas à criança apresentaram um incremento no grau de preenchimento da DNV. A completude do peso ao nascer e do sexo foi considerada adequada antes e após a descentralização do SINASC. Em relação às variáveis Índice de Apgar 10 minuto e Índice de Apagar 50 minuto, o preenchimento variou de não adequado (70\% a < $89,9 \%)$ a adequado $(90 \%-100 \%)$. O maior incremento no grau de preenchimento da DNV entre as variáveis relacionadas à criança foi verificado na variável raça/cor, considerando que este campo apresentou preenchimento crítico nos dois primeiros anos de implantação do SINASC (tabela 1).

Em relação ao preenchimento das características maternas com exceção para a idade materna, que manteve adequada para todos os portes desde a sua implantação, todas as outras variáveis apresentaram melhoria da completude após o ano 2000. O grau de instrução, estado civil, filhos nascidos vivos e filhos nascidos mortos apresentaram os piores preenchimentos nos dois primeiros anos, entre crítico (< de $70 \%$ ) e não adequado $(70 \%$ a $<89,9 \%)$. Entretanto, notouse um incremento importante no grau de preenchimento dessas variáveis, destacando-se os municípios de grande porte que apresentaram preenchimento adequado em todas elas. Nos municípios de pequeno e médio porte foram observados preenchimentos não adequados nas variáveis filhos nascidos vivos e filhos nascidos mortos.

Todas as variáveis relativas à gestação e ao parto apresentaram preenchimento adequado para todos os municípios, nos dois períodos avaliados.

Os resultados da análise das variáveis de acordo com a condição de gestão dos municípios apresentados na tabela 2 mostraram que os campos referentes ao peso ao nascer, sexo do recém nascido, idade da mãe, duração da gestação, tipo de gravidez, tipo de parto e consulta pré-natal apresentaram preenchimento adequado e pouco se modificaram nos períodos. Como observado na tabela 1 , verificou-se incremento importante no grau de preenchimento das variáveis raça/cor, grau de instrução, estado civil, filhos nascidos mortos e filhos nascidos vivos. Considerando as variáveis filhos nascidos vivos e filhos nascidos mortos, o preenchimento continua não adequado entre os municípios avaliados, independente da condição de habilitação no SUS. E a completude da variável raça/ cor ainda é inadequada entre os municípios na GPSM. As variáveis Apgar $10 \mathrm{mi}-$ nuto e Apgar 50 minuto apresentaramse estáveis nos dois períodos, com melhor preenchimento no conjunto dos municípios habilitados na GPSM.

\section{DISCUSSÃO}

Embora esse estudo não retrate a qualidade da informação do SINASC na perspectiva do seu processo, foi observado que a descentralização do Sistema de Informação, no conjunto dos municípios avaliados, independente do porte populacional e da condição de habilitação favoreceu o aprimoramento do preenchimento das informações da DNV, corroborando outros estudos em diferentes locais do país ${ }^{10-12,22-24}$.

Pressupõe-se que a gestão municipal imbuída do poder de avaliar, planejar e decidir ações buscou monitorar as informações sobre os nascidos vivos em função do contexto e dos interesses, valorizando o uso da informação em saúde. Por sua vez, o processo de descentralização das políticas governamentais de saúde tem produzido avanços com a constituição permanente de negociações, pactuações e arcabouço jurídico. Considerando as pactuações de indicadores materno-infantil e de cobertura na atenção básica e vigilância em saúde, exigiu-se dos gestores maior proximidade com o SINASC e com a fonte de captação dos eventos, implicando o monitoramento da coleta de dados ${ }^{13}$. Além disso, uma possível explicação é que nesse processo, houve um avanço na política de informação e informática em saúde ${ }^{3}$.

Entre as variáveis avaliadas, destacam-se as relativas ao peso ao nascer, sexo do recém nascido, idade da mãe e 
todos os campos relativos à gestação e ao parto (duração da gestação, número de consultas de pré-natal, tipo de gravidez e tipo de parto) que apresentaram as melhores completudes, mantendo-se adequadas nos períodos. Essas informações estão disponíveis nos prontuários do recém-nascido e da puérpera ou no cartão da gestante, o que facilita o registro do dado ${ }^{9}$.

Em relação às variáveis Apgar $1^{\circ}$ minuto e Apgar 50 minuto, observou-se que o grau de preenchimento variou entre não adequado e adequado ao longo dos anos. Os melhores resultados foram observados no conjunto dos municípios habilitados na GPSM. Costa e Frias ${ }^{12}$ questionam a fidedignidade dessas informações, uma vez que os dados preenchidos por profissionais não habilitados - aqueles que não têm conhecimento técnico para atribuir o valor referente às condições de vitalidade da criança no 10 e $5^{\circ}$ minutos - tendem a não ser preenchidos ou podem ser preenchidos com vício, repetindo-se o mesmo valor.

Os campos da DNV relacionados à raça/cor da criança, grau de instrução materna, estado civil, filhos nascidos vivos e filhos nascidos mortos apresentaram a maior variação de completude. Inclusive, nos municípios avaliados de pequeno e médio porte ainda se verificam preenchimentos não adequados nas variáveis filhos nascidos vivos e filhos nascidos mortos. Mello Jorge et al. ${ }^{6}$ já demonstraram o mesmo nos primeiros estudos de avaliação do SINASC, e problemas com essas variáveis ainda são observados em estudos mais recentes ${ }^{10-}$ 12,24. É possível que a incompletude dos registros dessas variáveis decorra de algumas questões como: deficiências ligadas à carência de recursos humanos qualificado; ausência de envolvimento constante dos diferentes profissionais que lidam com a informação em saúde, inclusive os gestores; falta de conhecimento do profissional responsável pelo preenchimento da DNV; ausência de informações nos prontuários da puérpera e do recém-nascido, até o desconhecimento de certas informações pelos acompanhantes e subjetividade envolvida na definição das categorias das variáveis, principalmente da raça/cor do recémnascido ${ }^{12,24-25}$

Os resultados deste estudo foram semelhantes à pesquisa realizada em estado do nordeste ${ }^{12}$, que mesmo utilizando metodologias diferentes, expressam evidências em relação à qualidade da informação do SINASC. Costa \& Frias $^{12}$ ao avaliar a evolução histórica da completude da DNV em Pernanbuco, entre 1996 e 2005, verificaram incremento importante no percentual de municípios avaliados com preenchimento excelente, destacando-se as variáveis raça/cor, escolaridade materna, estado civil materno e número de consultas pré-natal. Relatam que infelizmente, em algumas localidades, incompletudes ainda são verificadas nas variáveis número de fiIhos nascidos vivos, número de filhos nascidos mortos, Apgar $1^{\circ}$ minuto e Apgar $5^{\circ}$ minuto.

Em outro estudo, Mello Jorge et al. ${ }^{10}$, ao analisar a qualidade das estatísticas vitais brasileiras desde a concepção e implantação do SINASC, verificaram que as informações da DNV vêm melhorando acentuadamente, tanto a cobertura quanto a qualidade de seus dados. Relatam que as maiores incompletudes se devem aos campos das variáveis cor/raça da criança, Apgar $1^{\circ}$ minuto e Apgar $5^{\circ} \mathrm{mi}-$ nuto, escolaridade materna, filhos nascidos vivos e filhos nascidos mortos.

Romero \& Cunha ${ }^{11}$ reconheceram nas unidades federadas em 2002, diferenças no percentual de incompletude da DNV. Ao utilizar as variáveis em branco juntamente com as variáveis ignoradas como unidade de análise, as autoras observaram que as variáveis idade materna, sexo do recém- nascido, estado civil, escolaridade e peso ao nascer apresentaram de boa a excelente completude, enquanto os campos relativos à história reprodutiva materna, filhos tidos nascidos vivos e filhos nascidos mortos apresentaram preenchimento de ruim a regular.

Em Minas Gerais, no ano 2000, Souza ${ }^{16}$ apontou incompletudes maiores de $10 \%$ nas variáveis número de filhos vivos, número de filhos mortos, raça/cor, Apgar $1^{\circ}$ minuto e Apgar $5^{\circ}$ minuto. 
Estudo recente realizado em Tere$\operatorname{sina}^{24}$ apontou tendência de aprimoramento da qualidade do preenchimento da DNV, com bom preenchimento do formulário, exceto para a variável filhos tidos mortos que apresentou incompletude entre $10 \%$ e $29,9 \%$.

O presente estudo mostrou o aprimoramento da qualidade da informação do SINASC após sua a descentralização; entretanto, são necessários estudos de avaliação relacionados à estrutura, ao processo e ao impacto das informações

\section{REFERÊNCIAS}

1. Vieira da Silva LM. Avaliação do processo de descentralização das ações de saúde. Ciênc Saúde Coletiva. 1999; 4(2):331-339.

2. Ferla $A A$, Leal MB, Pinheiro, R. Um olhar sobre práticas avaliativas em descentralização do sistema de saúde: construindo uma abordagem analítica para atuação em rede. In: Pinheiro R, Mattos RA. Gestão em Redes. Práticas de Avaliação, formação e participação na Saúde. Rio de Janeiro: CEPESC; 2006.

3. Brasil. Ministério da Saúde. Portaria No 116, de 11 de fevereiro de 2009. Regulamenta a coleta de dados, fluxo e periodicidade de envio das informações sobre óbitos e nascidos vivos para os Sistemas de Informação em Saúde sob a gestão da Secretaria de Vigilância em Saúde. Ministério da Saúde, Secretaria de vigilância em Saúde; 2009.

4. Branco MA. Informação e Saúde: uma ciência e suas políticas em uma nova era. Rio de Janeiro: Fiocruz; 2006.

5. Brasil. Fundação Nacional de Saúde. Manual de instruções para o preenchimento da declaração de nascido vivo. Brasília: Centro de Documentação do Ministério da Saúde; 2001.

6. Mello-Jorge MHP, Gotlieb SL, Sabolli ML, Almeida MF, Latorre MR. O Sistema de Informação sobre Nascidos Vivos: primeira avaliação dos dados brasileiros. Inf Epidemiol SUS. 1996; 2:15-48. geradas pelo Sistema. Somente dessa maneira será possível verificar se essas informações de qualidade são utilizadas nos processos de monitoramento, avaliação e decisão da assistência à saúde da criança e da mulher.

Agradecimentos: À Secretaria de Estado de Saúde de Minas Gerais

Financiamento: Fundação de Amparo à Pesquisa do Estado de Minas Gerais - FAPEMIG; Fundação Oswaldo CruzFiocruz.

7. Frias PG, Pereira DMH, Vidal AS, Lira PIC. Avaliação da cobertura do Sistema de Informações sobre Nascidos Vivos e a contribuição das fontes potenciais de notificação do nascimento em dois municípios de Pernambuco, Brasil. Epidemiol e Serviços de Saúde. 2007; 16(2): 93101.

8. Silva AAM, Ribeiro VS, Borba Júnior AF, Coimbra LC, Silva RA. Avaliação da qualidade dos dados do sistema de informações sobre nascidos vivos em 1997 - 1998. Rev Saúde Pública. 2001; 35(6): 508-514.

9. Theme Filha MM, Gama SGN, Cunha CB, Leal MC. Confiabilidade do Sistema de Informações sobre Nascidos Vivos Hospitalares no Município do Rio de Janeiro, 1999-2001. Cad Saúde Pública. 2004; 20 Suppl 1:83-91.

10. Mello-Jorge MHP, Laurenti R, Gottlieb SLD. Análise da qualidade das estatísticas vitais brasileiras: a experiência de implantação do SIM e do SINASC. Cienc Saúde Coletiva. 2007; 12(3):643-54.

11. Romero ED, Cunha CB. Avaliação da qualidade das variáveis epidemiológicas e demográficas do Sistema de Informações sobre Nascidos Vivos, 2002. Cad Saúde Pública. 2007; 23(3):701-714.

12. Costa JMBS, Frias PG. Avaliação da completitude das variáveis da Declaração de Nascido Vivo de residentes em Pernambuco, Brasil, 1996 a 2005. Cad Saúde Pública. 2009; 25(3): 613-624. 
13. Almeida MF, Alencar GP. Informações em Saúde: necessidade de introdução de mecanismos de gerenciamento dos sistemas. Inf Epidemiol SUS. 2000; 4:241-249.

14. Minas Gerais. Superintendência de Epidemiologia. Análise de situação de saúde de Minas Gerais. SES/MG; 2007.

15. Drumond EF, Machado CJ, França E. Subnotificação de nascidos vivos: procedimentos de mensuração a partir do Sistema de Informação Hospitalar. Rev Saúde Pública. 2008; 42(1): 55-63.

16. Souza LM. Avaliação do Sistema de Informação sobre Nascidos Vivos SINASC, Minas Gerais e Mesoregiões, 2000 (Dissertação de mestrado). Belo Horizonte (MG): CEDEPLAR/FACE/ UFMG; 2004. 112 p.

17. Hartz ZM, Vieira da Silva LM (Orgs.). Avaliação em Saúde: dos modelos teóricos à prática na avaliação de programas e sistemas de saúde. Rio de Janeiro: Fiocruz; 2005.

18. Contandriopoulos AP, Champagne F, Denis JL, Pineaut RA. Avaliação na área da saúde: conceitos e métodos. In: Hartz ZMA (Org.). Avaliação em saúde: dos modelos conceituais à prática na implantação de programas. Rio de Janeiro: Fiocruz; 1997.

19. Minas Gerais. Plano Diretor de Regionalização de Minas Gerais. Secretaria de Estado de Saúde de Minas Gerais, 2010. [Acesso em: $29 \mathrm{dez}$ 2010]. Disponível em: http:// w w w. s a u d e.m g.gov. br / politicas_de_saude/plano-diretor-deregionalizacao-pdr

20. Brasil. Ministério da Saúde. Banco de dados dos sistemas de informação sobre mortalidade (SIM) e nascidos vivos (SINASC) 1998 a 2004 (CDROM). Brasília: MS/SVS; 2006.

21. Brasil. Ministério da Saúde. Banco de dados dos sistemas de informação sobre mortalidade (SIM) e nascidos vivos (SINASC), 1999 a 2005 (CDROM). Brasília: MS/SVS; 2007.

22. Barbuscia DM, Rodrigues-Júnior AL. Completude da informação nas Declarações de Nascido Vivo e nas Declarações de Óbito, neonatal precoce e fetal, da região de Ribeirão Preto, São PauIo, Brasil, 2000-2007. Cad. Saúde Pública. 2011; 27(6):1192-1200.

23. Silva GFS, Aidar T, Mathias TAF. Qualidade do Sistema de Informações de Nascidos Vivos no Estado do Paraná, 2000 a 2005. Rev Esc Enferm USP. 2011; 45(1):79-86.

24. Mascarenhas MDM, Gomes KRO. Confiabilidade dos dados do Sistema de Informações sobre Nascidos Vivos em Teresina, Estado do Piauí, Brasil 2002. Cienc Saúde Coletiva. 2011; 16(1):1233-1239.

25. Almeida MF, Alencar GP, França Junior I, Novaes HMD, Siqueira AAF, Scchoeps $D$, et al. Validade das informações das declarações de nascidos vivos com base em estudo de caso-controle. Cad Saúde Pública. 2006; 22(3):1-9. 